\title{
SK 1: A possible case of triggered star formation in perseus
}

\author{
Miriam Rengel $^{1}$, Klaus Hodapp ${ }^{2}$ \\ and Jochen Eislöffel ${ }^{3}$ \\ ${ }^{1}$ Max Planck Institute for Solar System Research, Katlenburg-Lindau, 37191, Germany \\ email: rengel@mps.mpg.de \\ ${ }^{2}$ Institute for Astronomy, 640 N. A'hookup Place, Hilo, HI 96720 \\ email: hodapp@ifa.hawaii.edu \\ ${ }^{3}$ Thüringer Landessternwarte Tautenburg, Sternwarte 5, 07778 Tautenburg, Germany \\ email: jochen@tls-tautenburg.de
}

\begin{abstract}
According to a triggered star formation scenario (e.g. Martin-Pintado \& Cernicharo 1987) outflows powered by young stellar objects shape the molecular clouds, can dig cavities, and trigger new star formation. NGC 1333 is an active site of low- and intermediate star formation in Perseus and is a suggested site of self-regulated star formation (Norman \& Silk 1980). Therefore it is a suitable target for a study of triggered star formation (e.g. Sandell \& Knee 2001, SK 1). On the other hand, continuum sub-mm observations of star forming regions can detect dust thermal emission of embedded sources (which drive outflows), and further detailed structures.

Within the framework of our wide-field mapping of star formation regions in the Perseus and Orion molecular clouds using SCUBA at 850 and $450 \mu \mathrm{m}$, we mapped NCG 1333 with an area of around $14^{\prime} \times 21^{\prime}$. The maps show more structure than the previous maps of the region observed in sub-mm. We have unveiled the known embedded SK 1 source (in the dust shell of the SSV 13 ridge) and detailed structure of the region, among some other young protostars.

In agreement with the SK 1 observations, our map of the region shows lumpy filaments and shells/cavities that seem to be created by outflows. The measured mass of SK $1\left(\sim 0.07 \mathrm{M}_{\odot}\right)$ is much less than its virial mass $\left(\sim 0.2-1 \mathrm{M}_{\odot}\right)$. Our observations support the idea of SK 1 as an event triggered by outflow-driven shells in NGC 1333 (induced by an increase in gas pressure and density due to radiation pressure from the stellar winds that have presumably created the dust shell). This kind of evidences provides a more thorough understanding of the star formation regulation processes.
\end{abstract}

Keywords. stars: formation, stars: individual, radio continuum: stars, ISM: jets and outflows, ISM: clouds.

\section{Introduction}

When an area of active or recent star formation is found, a question can be raised: is the star formation there happening spontaneously or is it being triggered? Places where there is no apparent trigger would be a possible evidence of its spontaneous nature, but in areas where there are indications of triggered star formation the determination of the possible causes is not easy. Because star forming areas contain populations of young stellar objects (YSOs), the mentioned question can be addressed by studying the interactions between components of the YSOs and their surrounding Interstellar Medium (ISM).

Powerful outflows are associated with strong accretion activity of the earliest protostellar phase (Class 0 stage) in the formation of a low-mass star. The Class 0 phase was identified by André, Ward-Thompson \& Barsony 1993, and consists of a central protostellar 
object, surrounded by an infalling envelope, and a flattened accretion disk. The later SED classes (1,2 and 3, e.g. Lada 1987) are characterized by progressively diminishing accretion rates and consequently, less outflow power (Bontemps, André, Terebey, et al. 1996; Henriksen, André \& Bontemps 1997; Davis \& Eislöffel 1995).

The Class 0 phase is of special interest, not only because most of the characteristics of a future star are determined during this phase, but outflows can have a profound effect on the surrounding ISM: they shape the molecular clouds, can dig cavities, compress dust shells and trigger new star formation. Observations of this phase are, however, difficult for two reasons: first, the hot, near-stellar core of a Class 0 object is so heavily obscured $\left(\mathrm{A}_{V} \approx 500 \mathrm{mag}\right)$ as to make the object undetectable up into the mid-IR. Second, the Class 0 phase is of short duration; presumably of order of a few $10^{5}$ yr (Visser, Richer \& Chandler 2002). Therefore, only a small number of these objects has been found to allow detailed studies. Nevertheless, continuum sub-mm observations of these sources can detect dust thermal emission of the circumstellar envelopes and provide a powerful tool for constraining the distribution of matter in Class 0 objects (Adams 1991).

As part of a more extensive study of star-forming regions and of the physical structure and processes in Class 0 sources (Rengel 2004), we report here the possibility of triggered star formation by outflows driven by YSOs and discuss evidence for this triggering.

\section{Imaging of the molecular cloud NGC 1333 and results}

\subsection{Target selection}

Together with the regions L1448, L1455, HH211 (in Perseus) and L1634 and L1641 N (in Orion), we map NCG 1333 at 850 and $450 \mu \mathrm{m}$ using the Submillimetre Common User Bolometer Array (SCUBA) camera at the James Clerk Maxwell Telescope (JCMT). NGC 1333 is an active site of low- and intermediate star formation in Perseus and is a suggested site of self-regulated star formation (Norman \& Silk 1980). It has been observed in the sub-mm by Looney, Mundy \& Welch 2000, Sandell \& Knee 2001, and Chini, WardThompson, Kirk, et al. 2001. NGC 1333 IRAS 2 (Jennings; Cameron, Cudlip, et al. 1987) is located at the edge of the large cavity (Langer, Castets \& Lefloch 1996). It has been resolved into three sources: 2A and 2B, detected by several authors (Sandell, Knee, Aspin, et al. 1994; Blake, Sandell, van Dishoeck, et al. 1995; Lefloch, Castets, Cernicharo, et al. 1998; Rodríguez, Anglada \& Curiel 1999; Looney, Mundy \& Welch 2000; Sandell \& Knee 2001; Jørgensen, Hogerheijde, van Dishoeck, et al. 2004), and 2C, detected by Sandell \& Knee (2001). IRAS 2 A and B are Class 0 candidates (Sandell \& Knee 2001; Motte \& André 2001). Observations of IRAS 2 (A,C) in mid-IR are reported by Rebull, Cole, Stapelfeltd, et al. 2003.

\subsection{Observations}

NGC 1333 was mapped here with an area of $14^{\prime} \times 21^{\prime}$ with both jiggle and scan maps. Data reduction treatment is described in Rengel (2004). These new maps show more structure than previous sub-mm maps of this region. We include SSV 13, south areas, the region surrounded IRAS 1 and NGC $1333 \mathrm{~S}$. This later region was first noted by Rengel, Froebrich, Hodapp, et al. (2002), further discussed by Rengel (2004) and independently discovered by Young, Shirley, Evans, et al. (2003). Hodapp, Bally, Eislöffel, et al. (2005) discussed a subset of the sub-mm data presented here in their relation to NIR observations and pointed out that the driving source of the system HH343A-F-HH340B was likely associated with the easternmost clump in NGC 1333 S. Newly identified structure introduced by Rengel, Hodapp \& Eislöffel (2005) strengthen the tentative conclusion reached by Hodapp, Bally, Eislöffel, et al. (2005) that NGC 1333 S is the site of secondary, 

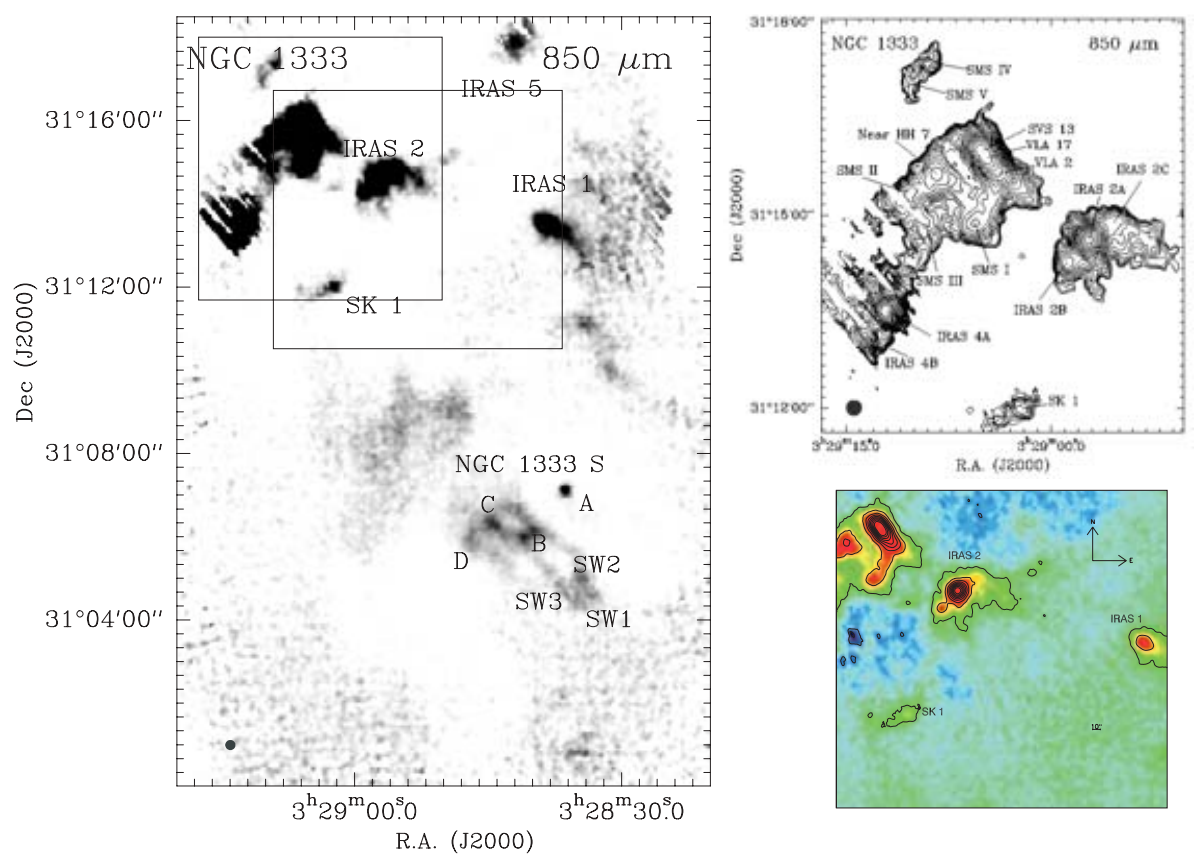

Figure 1. The $850 \mu \mathrm{m}$ deep field map of NGC 1333 (left) and the $850 \mu \mathrm{m}$ contour map of the SSV 13 area in NGC 1333 (upper right) (left box in left figure). Contour levels are in log scale with step size 1.25 from $0.0002 \mathrm{Jy}_{\text {beam }}{ }^{-1}$. The filled circles in the bottom left indicate the main beam size. Down right image shows the deep field map of the region containing SK 1.

low-mass star formation triggered by the powerful IRAS 1-9 protostars about 1 pc north of this region.

Furthermore, SK 1 is an embedded source in the dust shell south of the SVS 13 ridge, with an emission (east) detected in the $850 \mu \mathrm{m}$ map. We unveiled it, and found several dust ridges and shells formed by outflows in the region, among further detailed structure and some other YSOs (Fig. 1). Sandell \& Knee (2001) suggest that SK 1 appears to be a case of triggered star formation by outflow-driven shells. Further details of the mapped region are given in Rengel (2004) and Rengel, Hodapp \& Eislöffel (2006).

\section{Deriving physical parameters}

We estimate the gas and dust masses for the sample from the dust emission according to the mass equation of Hildebrand (1983). We find a mass of $3.7 \mathrm{M}_{\odot}$ for IRAS $2 \mathrm{~A}$ and $\sim 0.07 \mathrm{M}_{\odot}$ for SK 1 .

We infer the bolometric temperature and luminosity of IRAS 2A and SK 1 by constructing the complete SEDs (we combine the SCUBA fluxes with data from the literature in several wavelengths [Rengel, Froebrich, Wolf, et al. 2004]). Ages are further calculated following the Smith protostellar evolutionary scheme (Smith 1999, 2002). For IRAS 2 A and SK 1 , we find ages of 26 and $15 \times 10^{3} \mathrm{yr}$, respectively.

Within the framework of deriving physical parameters of the YSOs contained our widefield mapping of star formation regions, we also establish further physical conditions (e.g. temperature distribution, radius, and power-law index of the density) of some sources in the sample, including IRAS $2 \mathrm{~A}$. This is performed by using the radiative transfer code MC3D (Wolf, Henning \& Stecklum 1999). Further modeling details are given in Rengel (2004) and Rengel, Hodapp \& Eislöffel (2006). 


\section{SK 1: Suspected triggered star formation in NGC 1333}

Are the molecular outflows of IRAS 2 (Knee \& Sandell 2000; Jørgensen, Hogerheijde, Blake, et al. 2004) perhaps the main cause of the formation of SK 1? We report here some evidences that support the idea of modification and disruption of areas by the IRAS 2 outflows, and the formation of SK 1 as a result of the compressed dust shells/cavities.

First, our map of the region shows lumpy filaments and shells/cavities that seem to be created by outflows. Second, the measured mass of SK 1 is much less than its virial mass $\left(\sim 0.2-1 \mathrm{M}_{\odot}\right)$. This indicates that SK 1 is not in a stationary state, isolated and self-gravitationally bound. Third, regarding the star formation timing, a hard upper limit is placed on the age: IRAS $2 \mathrm{~A}$ is older than SK 1. Fourth: which one physical mechanism is responsible of the formation of SK 1 ? we rule out cluster dissipation as the main mechanism: if a standard cluster dispersion velocity of $1 \mathrm{~km} \mathrm{~s}^{-1}$ is assumed, SK 1 has not traveled far. "Collect-and-collapse" (CAC) and radiation-driven implosion (RDI) scenarios (Elmegreen \& Lada 1977; Oort \& Spitzer 1955) have been proposed as physical mechanisms for the star formation at the edge of an HII region. Timescales for the RDI mechanism are less than for the CAC scenarios, then it seems that this later one is the most supported. Fifth, IRAS 1 has a long tail pointing away from the HII region, which is an indication of direct interaction between the outflows and the gas and dust in the vicinity.

\section{Conclusion}

SK 1 provides an interesting study of interactions between outflows and surrounding ISM. In at least one case, we identify this protostellar source whose formation is likely to have been triggered by powerful outflow bow shocks. This kind of evidence provides a more thorough understanding of the star formation regulation processes. Further observations of molecular gas are necessary.

\section{Acknowledgements}

We thank the S237 organizers, and T. Jenness for the assistance with SURF.

\section{References}

Adams, F. C. 1991, ApJ 382, 544

André, P., Ward-Thompson, D. \& Barsony, M. 1993, ApJ 406, 122

Blake, G. A., Sandell, G., van Dishoeck, E. F., et al. 1995, ApJ 441, 689

Bontemps, S., André, P., Terebey, S. \& Cabrit, S. 1996, A $\mho A$ A 311, 858

Chini, R., Ward-Thompson, D., Kirk, J. M., et al. 2001, A\&A 369, 155

Davis, C. J. \& Eislöffel, J. 1995, A\&3A 300, 851

Elmegreen, B. G. \& Lada, C. J. 1977, ApJ 214, 725

Henriksen, R., André, P. \& Bontemps, S. 1997, A\& A 323, 549

Hildebrand, R. H. 1983, QJRAS 24, 267

Hodapp, K. W., Bally, J., Eislöffel J. \& Davis, C. J. 2005, AJ 129, 1580

Jennings, R. E., Cameron, D. H. M., Cudlip, W. \& Hirst, C. J. 1987, MNRAS 226, 461

Jørgensen, J. K., Hogerheijde, M. R., Blake, G. A., van Dishoeck, E. F., Mundy, L. G. \& Schöier, F. L. 2004, A\&A 415, 1021

Jørgensen, J. K., Hogerheijde, M. R., van Dishoeck, E. F., Blake, G. A. \& Schöier, F. L. 2004, AESA 413, 993

Knee, L. B. G. \& Sandell, G. 2000, A\&A 361, 671

Lada, C. J. 1987, in: M. Peimbert \& J. Jugaku (eds.), Star Forming Regions (Dordrecht: Kluwer), p. 1 
Langer, W. D., Castets, A. \& Lefloch, B. 1996, ApJ 471, 111

Lefloch, B., Castets, A., Cernicharo, J. \& Loinard, L. 1998, ApJ 504, L109

Looney, L. W., Mundy, L. G. \& Welch, W. J. 2000, ApJ 529, 477

Martin-Pintado, J. \& Cernicharo, J. 1987, A\& A 176, 27

Motte, F. \& André, P. 2001, A\&̊A 365, 440

Norman, C. \& Silk, J. 1980, ApJ 238, 158

Oort, J. H. \& Spitzer, L., Jr. 1955, ApJ 121, 6

Rebull, L. M., Cole, D. M., Stapelfeltd, K. R. \& Werner, M. W. 2003, AJ 125, 2568

Rengel, M. Froebrich, D., Hodapp, K. \& Eislöffel, J. 2002, in: J. F. Alves \& M. J McCaughrean (eds.), The Origins of Star and Planets: The VLT View (Berlin: Springer), CD-ROM

Rengel, M., Froebrich, D., Wolf, S. \& Eislöffel, J. 2004, BaltA 13, 449

Rengel, M. 2004, Ph.D. Thesis, Friedrich-Schiller Universität, Jena

Rengel, M., Hodapp, K. \& Eislöffel, J. 2005, AN 326, 631

Rengel, M., Hodapp, K. \& Eislöffel, J. 2006, A\&A submitted

Rodríguez, L. F., Anglada, G. \& Curiel, S. 1999, ApJS 125, 427

Sandell, G., Knee, L. B. G., Aspin, C., Robson, I. E. \& Russell, A. P. G. 1994, A\&A 285, L1

Sandell, G. \& Knee, L. B. G. 2001, ApJ 546, L49

Smith, M. D. 1999, ApSS 261, 169

Smith, M. D. 2002, in: J.F. Alves \& M.J McCaughrean (eds.), The Origins of Stars and Planets: The VLT View (Berlin: Springer), CD-ROM

Visser, A. E., Richer, J. S. \& Chandler, C. J. 2002, AJ 124, 2756

Wolf, S., Henning, Th. \& Stecklum, B. 1999, A\&A A 349, 839

Young, C. H., Shirley, Y. L., Evans, N. J., II \& Rawlings, J. M. C. 2003, ApJS 145, 111

\section{Discussion}

NAKAmURA: Could you make some comment on the physical conditions of SK1 before the outflow shock hit the core?

RENGEL: With our evolutionary models, and knowing the actual physical structure of SK1, we can go back to earlier stages (e.g., before the outflow shock hit the core) and calculate the physical conditions of SK1 (size, $\mathrm{T}_{\mathrm{bol}}, \mathrm{L}_{\mathrm{bol}}$, mass, etc.). As presumably SK1 is a very young protostar, it has more massive envelope, and it was cooler. 